\title{
Compact Wavelength-Selective Functions in Silicon-on-Insulator Photonic Wires
}

\author{
Wim Bogaerts, Member, IEEE, Pieter Dumon, Student Member, IEEE, Dries Van Thourhout, Member, IEEE, \\ Dirk Taillaert, Member, IEEE, Patrick Jaenen, Johan Wouters, Stephan Beckx, Vincent Wiaux, \\ and Roel G. Baets, Senior Member, IEEE
}

\begin{abstract}
We present a number of compact wavelength-selective elements implemented in silicon-on-insulator (SOI) photonic wires. These include arrayed waveguide gratings (AWGs), MachZehnder lattice filters (MZLFs), and ring resonators. The circuits were fabricated with deep UV lithography. We also address the sensitivity of photonic wires to phase noise by selectively broadening the waveguides, and demonstrate this in a compact AWG with $-20 \mathrm{~dB}$ crosstalk and an insertion loss of $2.2 \mathrm{~dB}$ for the center channels.
\end{abstract}

Index Terms-Arrayed waveguide grating (AWG), nanophotonics, photonic wire, ring resonator, silicon-on-insulator (SOI).

\section{INTRODUCTION}

$\mathbf{N}$ ANOPHOTONIC circuits are the most promising route toward large-scale photonic integration, combining many optical functions onto a single chip. This should lead, as in electronics, to a dramatic increase in functionality and a serious reduction in cost through efficient wafer-scale processes. However, today's commercially available devices integrate only a few functions onto a chip, because individual elements have a length scale from several hundred micrometers up to centimeters. These dimensions are mostly dictated by the low contrast in refractive index $n$ of the material, resulting in poorly confined waveguide modes that need a large bend radius. A higher index contrast improves the confinement, but then the waveguide core should be reduced in size to have a single-mode waveguide. For very high index contrast, as in semiconductor-to-air $(\Delta n>2)$ this results in so-called photonic wires with submicron core dimensions, which have to be fabricated with an accuracy in the range of $1-10 \mathrm{~nm}$. The silicon-on-insulator (SOI) material system is ideally suited for such nanophotonic waveguides. It consists of a thin layer of silicon $(n=3.45)$ on top of an oxide buffer layer $(n=1.45)$, resulting in a high index contrast in both vertical and lateral directions.

Manuscript received November 15, 2005; revised August 9, 2006. This work was supported in part by the European Union under Project IST-PICCO, Project IST-PICMOS, and the IST-ePIXnet Network of Excellence, and in part by the Belgian IAP-PHOTON Network Project. The work of W. Bogaerts was supported by the Flemish Fund for Scientific Research (FWO). The work of P. Dumon and D. Taillaert was supported by the Flemish Institute for the Industrial Advancement of Scientific and Technological Research (IWT).

W. Bogaerts, P. Dumon, D. Van Thourhout, D. Taillaert, and R. G. Baets are with the Department of Information Technology, Ghent UniversityInteruniversity Microelectronics Center, 9000 Ghent, Belgium (e-mail: wim.bogaerts@intec.ugent.be; baets@intec.rug.ac.be).

P. Jaenen, J. Wouters, S. Beckx, and V. Wiaux are with the Silicon Processing Technology Division, Interuniversity Microelectronics Center vzw., 3001 Leuven, Belgium.

Digital Object Identifier 10.1109/JSTQE.2006.884088
An undesirable consequence of this high index contrast is the increased sensitivity to perturbations, like scattering at roughness on the core-cladding interface [1]. In interferometric structures, the waveguides will be susceptible to phase fluctuations due to small changes in waveguide geometry. We therefore need high-quality fabrication tools. For this paper, we use deep UV lithography, as used for advanced complementary metal-oxide-semiconductor (CMOS) fabrication [2].

Current optical fiber links make use of wavelength-division multiplexing (WDM), in which data is multiplexed over many channels, each with its own carrier wavelength. However, at nodes these wavelength channels have to be combined or separated using wavelength-selective elements. These can be filters that select one or a few wavelength channels, which can be implemented as resonators or interferometers. Alternatively, one can use all-out (de)multiplexers that process all channels simultaneously. The often-used device today is the arrayed waveguide gratings (AWG), an optical phased array antenna with a wavelength-dependent phase delay. However, an AWG in today's low-index-contrast materials easily uses many square centimeters of chip area, making it difficult to add more functionality to these circuits. The use of nanophotonic waveguides can dramatically reduce the required real estate.

In this paper, we give an overview of a number of wavelength-selective functions implemented in SOI photonic wires. In Section II, we describe the fabrication process for SOI nanophotonic circuits and our method of characterization. Then, we briefly describe basic photonic wires in Section III. Section IV covers basic properties of wavelength-selective elements. We then discuss the experimental results for AWGs in Section V, Mach-Zehnder lattice filters (MZLFs) in Section VI, and ring resonators in Section VII.

\section{FABRICATION AND CHARACTERIZATION}

\section{A. Fabrication of SOI Waveguides}

For this paper, we use SOI wafers consisting of a 220-nm thin silicon layer $(n=3.45)$ on top of a $1-\mu \mathrm{m}$ oxide cladding layer ( $n=1.45)$ carried on a bare silicon wafer. The oxide thickness of $1 \mu \mathrm{m}$ is sufficient to reduce substrate leakage [3].

For this paper, we used the advanced CMOS research environment of the Interuniversity Microelectronics Center, Belgium. The structures are patterned with 248-nm deep UV lithography and directly etched into the top silicon layer with an ICPRIE etch, using the resist as the etch mask. The buried oxide is not etched. This fabrication process is described in detail in [2] and [3]. 


\section{B. Characterization}

We characterize the devices using transmission measurements. For incoupling and outcoupling, we use grating couplers that couple light directly from a broad ridge waveguide in and out of a standard single-mode fiber, which is positioned above the substrate at a $10^{\circ}$ angle [4], [5]. Coupling efficiency is $33 \%$ $(-5 \mathrm{~dB})$ per coupler. This technique does not require cleaved or polished facets, and can also be used with array connectors [6]. The fiber couplers used here couple just one fiber polarization into the waveguide transverse electric (TE) mode.

\section{Photonic Wire WaVEguides}

\section{A. Basic Properties}

Photonic wires are basically index-guiding optical waveguides with a submicron core and a high index contrast. With a core thickness of 220-nm, single-mode waveguides for $1.55 \mu \mathrm{m}$ should be narrower than $580 \mathrm{~nm}$. Because it is very difficult to make these waveguides polarization-independent, all our components are designed for a TE polarization only.

Photonic wires also have significantly more dispersion than conventional, low-contrast waveguides [7], although not as high as that of some photonic crystal waveguides [8]. This results in a high group index between $n_{\mathrm{g}}=4$ and 4.7 , depending on the waveguide width. In interferometric structures, a high group index (and, thus, narrow wires) are desirable, but narrow wires are more susceptible to phase noise, which can significantly degrade the performance of the device.

\section{B. Propagation Losses}

We have previously demonstrated propagation losses as low as $0.24 \mathrm{~dB} / \mathrm{mm}$ in straight photonic wires [2], [9]. Similar results have been reported by other groups [10], [11] with e-beam fabricated waveguides.

We have also characterized bend losses using a large spiral waveguides [inset in Fig. 1(b)]. We designed a statistically significant spread in both waveguide length (between 0.1 and $50 \mathrm{~mm}$ ) and number of bends (between 0 and 550). In Fig. 1(b), we plot the transmission for spirals with a $3-\mu \mathrm{m}$ bend radius as a function of the total length and the number of $90^{\circ}$ bends. The straight propagation loss and the excess loss per $90^{\circ}$ bend is given by the slope of a two-dimensional (2-D) fit. For this radius, the excess bend loss per $90^{\circ}$ bend is only $0.016 \mathrm{~dB}$.

As expected, the excess bend losses increases dramatically for sharper bends. The total loss of a bend is this excess loss plus the straight propagation loss along the length of the bend. The latter contribution increases with the bend radius. This is plotted in Fig. 1(b). For a 1- $\mu \mathrm{m}$ bend radius, the excess loss of over $0.1 \mathrm{~dB} / 90^{\circ}$ dominates, while for a $5-\mu \mathrm{m}$ bend radius, the excess loss is of the same order as the propagation loss, resulting in a total loss of only $0.01 \mathrm{~dB} / 90^{\circ}$ bend. Similar number for bend losses have been reported in the literature [12], although measured on much fewer bends.

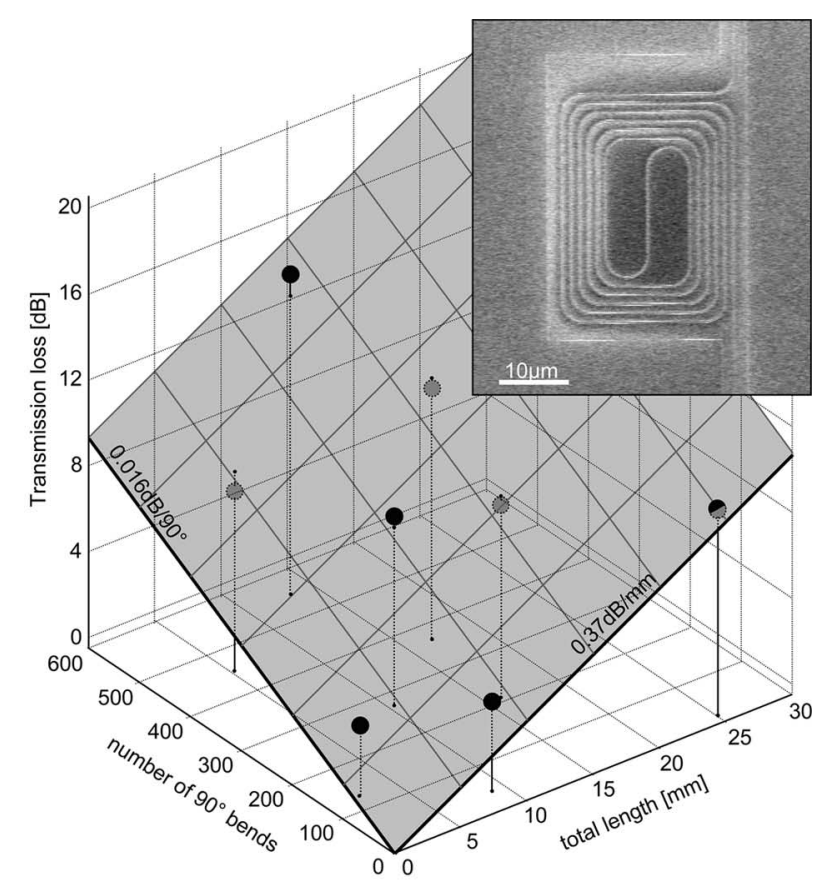

(a)

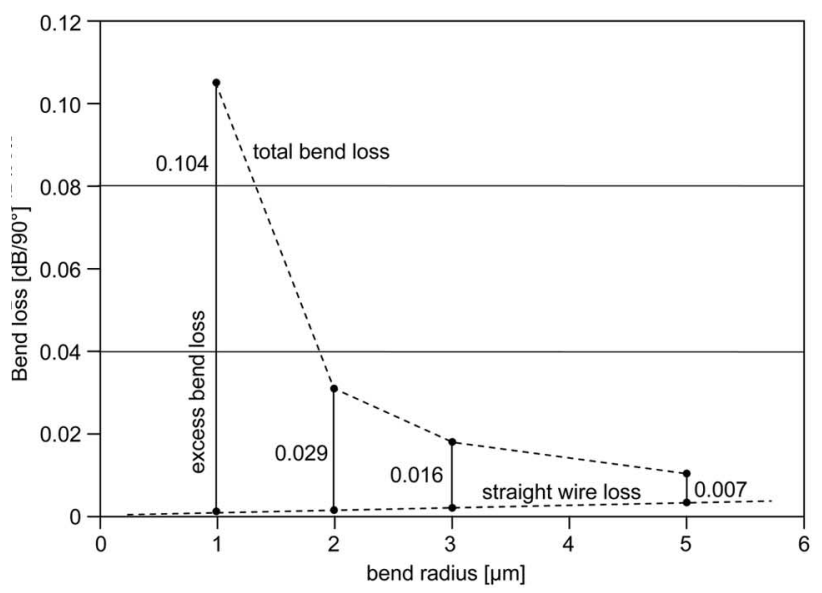

(b)

Fig. 1. Bend losses in photonic wires. (a) Transmission loss for a set of spiral waveguide (inset) with $3-\mu \mathrm{m}$ bend radius as a function of the number of $90^{\circ}$ bends and the total waveguide length. The slope of the fitted plane along the axes is the straight waveguide loss and the excess bend loss. (b) Bend loss per $90^{\circ}$ bend as a function of the bend radius. The loss of a straight waveguide of equal length is also plotted with the values for the excess loss per bend.

\section{WAVELENGTH-SELECTIVE ELEMENTS}

With photonic wires, a dramatic reduction of passive wavelength-selective elements can be obtained. Small resonant cavities can be used as channel drop filters with a large free spectral range (FSR), to be used in systems with many wavelength channels. However, in many applications, one operates in a more limited wavelength range $(<30 \mathrm{~nm})$ with standard WDM frequency channel spacings as $100 \mathrm{GHz}(0.8 \mathrm{~nm})$ or 200 $\mathrm{GHz}(1.6 \mathrm{~nm})$. In these cases, sometimes a rather small FSR is needed. As the delay line length needed in interferometric devices is inversely proportional to the FSR, this can result in delay lengths that are much larger than the bend radius, as in [6]. 


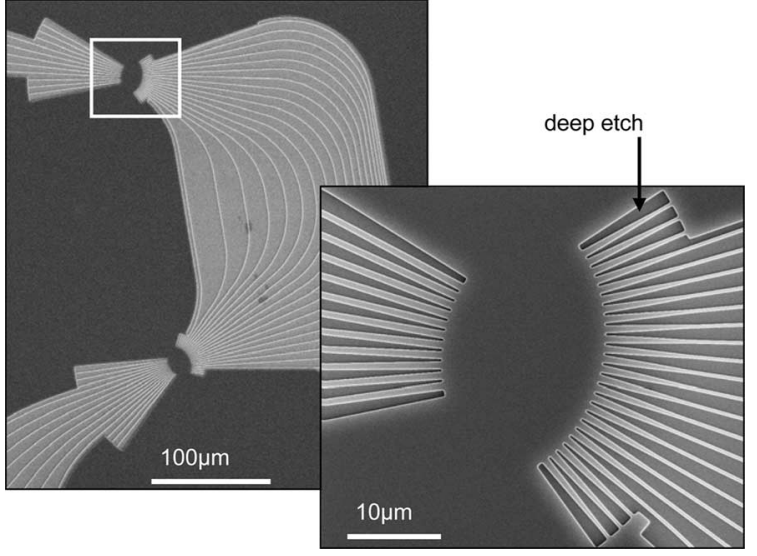

Fig. 2. AWG with $8 \times 400 \mathrm{GHz}$ channels.

This partially cancels out some advantages of using sharp bends, unless one starts folding the delay lines. Without such folding, an AWG with just a few closely spaced channels (and thus a small FSR) will turn out to be quite large compared to a similar AWG with more channels, even if much more grating arms are required. When the differential delay length is of the same order of magnitude as the bend radius and is three to four times larger than the minimal spacing between the waveguides, a very compact stacking of the delay lines is possible. As an example, a circular eight-channel AWG with $400 \mathrm{GHz}(3.2 \mathrm{~nm})$ channel spacing has an FSR of $25.6 \mathrm{~nm}$, and it will inherently be larger than a device with the same number of channels with a two times larger channel spacing.

A notable exception to this is an AWG-based (de)multiplexer, where only the center channels are used to obtain low insertion loss nonuniformity. In such an AWG, the FSR is much larger than the channel spacing times the number of used channels, as only the center channels are actually used. This implies a small path length difference between arms, allowing a close packing. For a given FSR, the size is then largely determined by the number of arms, which is typically two to three times the number of wavelength channels (including the channels that are not used).

Therefore, the actual reduction in size of a device is not just limited by the waveguide itself, but also by the desired FSR, fixing the delay lengths needed in interferometric devices, and the performance targets set.

\section{AWGs}

AWGs are currently the most popular integrated devices for multiplexing and demultiplexing multiple wavelength channels. Photonic wires with short bend radii and high group index can dramatically reduce these dimensions. In 2004, we have demonstrated an eight-channel AWG in SOI, which has a footprint of only $380 \mu \mathrm{m} \times 290 \mu \mathrm{m} \approx 0.1 \mathrm{~mm}^{2}$ or about $0.1 \mathrm{~mm}^{2}$ [13]. This device is shown in Fig. 2(a). However, this device has a number of limitations. The layout is designed with the same design rules as for low-contrast AWGs, which are not really compatible with the very sharp bends possible in photonic wires. Also, the high refractive index contrast of the wires makes it dif-

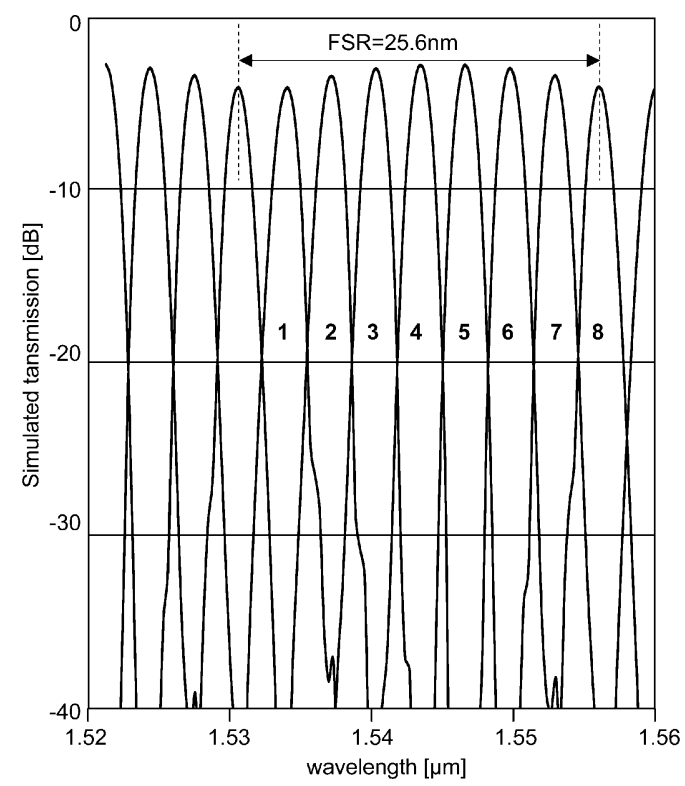

(a)

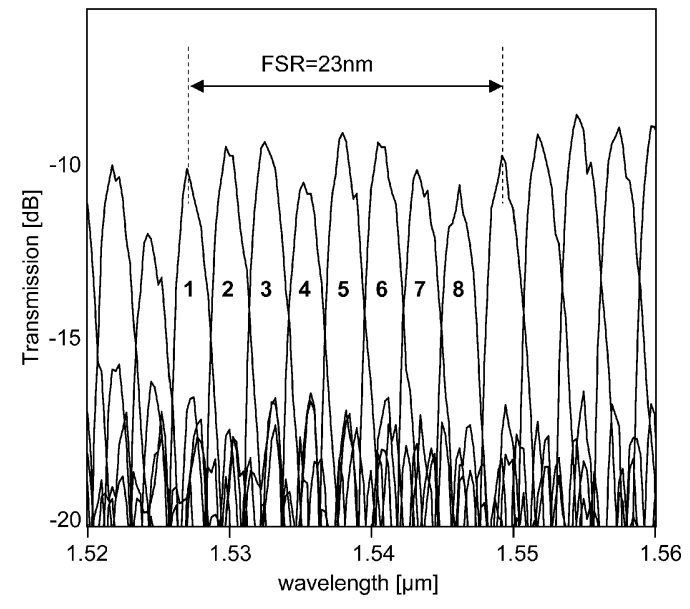

(b)

Fig. 3. AWG with $8 \times 400 \mathrm{GHz}$ channels. (a) Simulated transmission spectrum from the eight output ports. (b) Measured transmission spectrum.

ficult to implement good "free-space" star couplers, as there is a significant vertical mode mismatch between the photonic wire waveguide mode and the slab modes in the star coupler. This can result in unwanted reflections, which increase the overall insertion loss of the device. Also, the delay lines implemented in narrow photonic wires are prone to phase noise due to small fluctuations in waveguide geometry. The device in Fig. 2 has a designed channel spacing of $3.2 \mathrm{~nm}(400 \mathrm{GHz})$ and an FSR of $25.6 \mathrm{~nm}$.

We simulated this design using 2-D propagation in the slab regions of the star couplers [14]. The result is plotted in Fig. 3(a). While the general crosstalk is quite low, there is still a significant nearest neighbor crosstalk because of the broad channel transmission peaks. In this simulation, we did not incorporate phase noise due to waveguide roughness and irregularities.

The measured transfer from the input port to the eight output waveguides is plotted in Fig. 3(b). The transmission is normalized to the transmission of a simple photonic wire; hence, the 
scale represents only the losses in the AWG. The on-chip insertion loss of the AWG is approximately $8 \mathrm{~dB}$. However, the main limitation is the high crosstalk level limited to values around $-7 \mathrm{~dB}$, which is clearly not sufficient for practical applications. The main part of this crosstalk can be attributed to the phase noise generated in the waveguide arms. The shift in the center wavelength and the FSR between the simulated and the fabricated device can be attributed to deviation of the wire geometry with respect to the design. This could be compensated by global tuning of the device, e.g., thermal tuning.

In [15], an AWG fabricated using SOI wires was presented. The authors optimized the devices for compactness and demonstrated a $110 \mu \mathrm{m} \times 93 \mu \mathrm{m}$ device with a $6-\mathrm{nm}$ channel spacing and a $900-n m$ FSR. Also, the crosstalk was significantly worse, at a level of $-5 \mathrm{~dB}$. Another example with a smaller footprint, but still with a similarly large FSR, was demonstrated in [16]. The reported insertion loss was very low, but it was normalized to the average propagation loss in a grating arm, which can be significant given the reported losses of $12 \mathrm{~dB} / \mathrm{mm}$.

We show an improved device in Fig. 4. A more flexible layout has been used, which made sharp bends and close spacing of the arms possible, taking advantage of the tight confinement of photonic wire waveguides. To reduce the crosstalk due to phase noise in the narrow photonic wires, we have broadened the waveguides in the long straight sections of the grating arms. These sections are no longer single-mode, but as there are no bends in these sections, the coupling to higher order modes should be very small. Also, any light in higher order modes will be filtered out as the wire is reduced in width near a bend. However, as the broadened wires have a different group index as the narrow wires, the required physical delay length will be larger, resulting in a slightly larger device than when no broadened wire has been used.

To address the high insertion loss in the star couplers, we reduced the reflections at the waveguide apertures by using a two-step etch process, where the waveguides near the star coupler are etched only 70-nm deep, compared to the 220-nm etch depth of the photonic wires. This effectively reduces the lateral index contrast near the star coupler [17]. This is shown in Fig. 4. We used an adiabatic transition between the deeply etched and shallow waveguide, which requires a length of 15-30 $\mu \mathrm{m}$, depending on the parameters of the star coupler. Of course, this will also add to the footprint of the device. The star couplers can be further optimized by changing the geometry of the access waveguides, as in [16].

Fig. 4 shows a 16-channel AWG with 36 arms, a designed channel spacing of $1.6 \mathrm{~nm}(200 \mathrm{GHz})$, and a designed FSR of $25.6 \mathrm{~nm}$. The experimental values differ slightly, which can again be attributed to the waveguide geometry. The FSR is the same as in the previous example, as is the footprint $\left(500 \mu \mathrm{m} \times 200 \mu \mathrm{m}=0.1 \mathrm{~mm}^{2}\right)$. However, the combination of a rectangular geometry and a bend radius of only $4 \mu \mathrm{m}$ supports almost twice the number of arms in the same area, allowing for twice the number of channels with half the channel spacing.

These modifications to the AWG design lead to an improved transmission characteristic. Fig. 5(a) shows the simulated transmission of the 16 output ports as a function of the wavelength

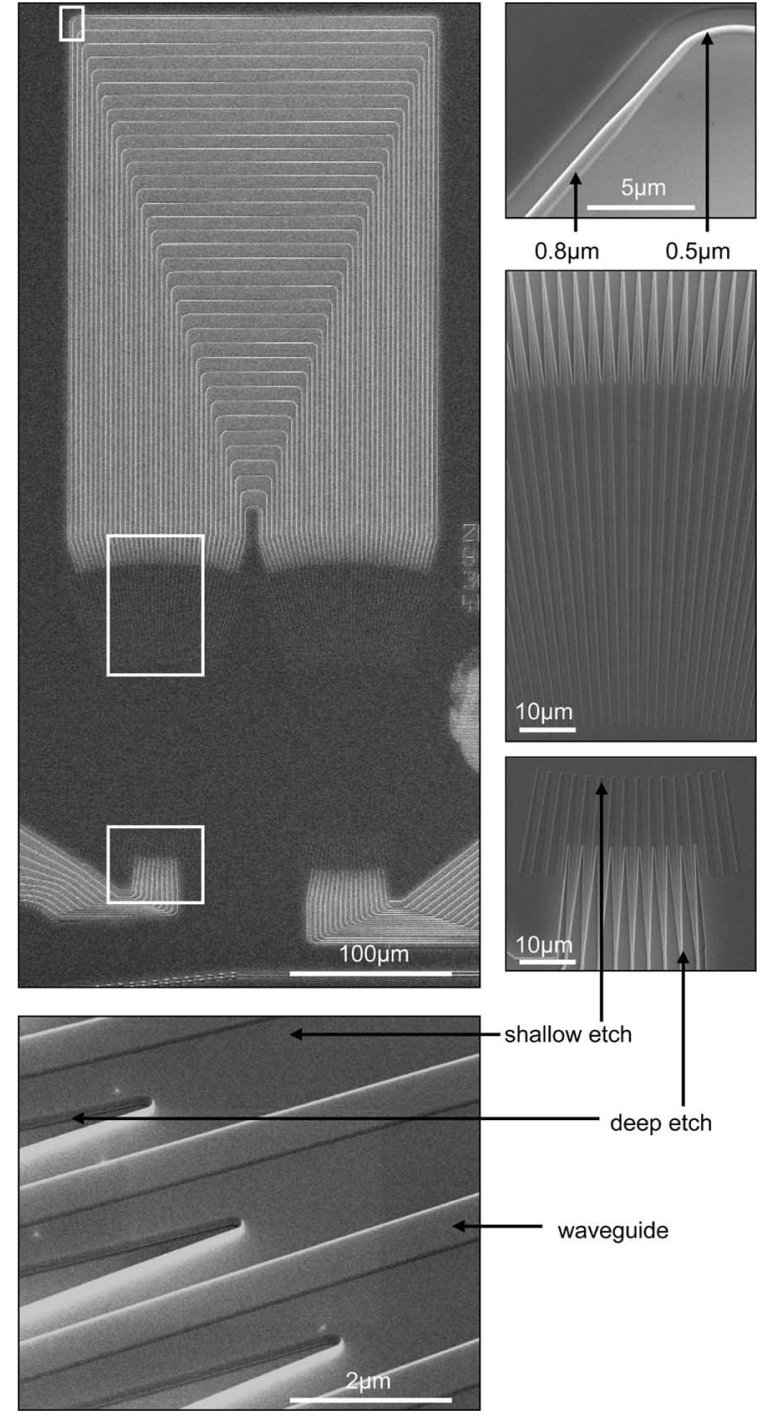

Fig. 4. AWG with $16 \times 200 \mathrm{GHz}$ channels. Details show broadened photonic wires in straight sections and the two-step star coupler with shallow etched waveguides.

in the central input ports. Simulated insertion loss is less than $-1 \mathrm{~dB}$, which can be attributed to the shallow-etch star coupler. As expected, there is a $3-\mathrm{dB}$ nonuniformity between the center and the extreme outputs. Without phase noise, the general crosstalk is $-35 \mathrm{~dB}$, while the nearest neighbor crosstalk is $-28 \mathrm{~dB}$.

The measured transmission characteristic is shown in Fig. 5. Again, the transmission is normalized to a simple photonic wire. The device has a general crosstalk between -17 and $-20 \mathrm{~dB}$. The nearest neighbor crosstalk is even lower. Due to the shallowetch star couplers, the insertion loss has improved considerably, being only $-2.2 \mathrm{~dB}$ in the center output ports. Again, the nonuniformity between the center and the outer ports is $3 \mathrm{~dB}$.

In this design, phase noise is still believed to be the dominant cause of crosstalk. To reduce the phase noise even further, one should resort to better fabrication processes, or ultimately to active tuning of the grating arms. 


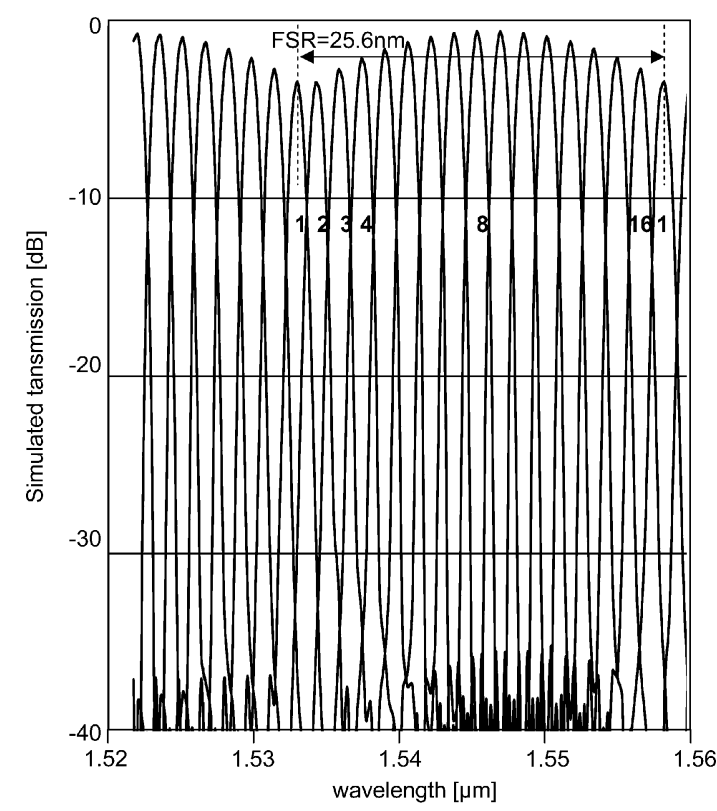

(a)

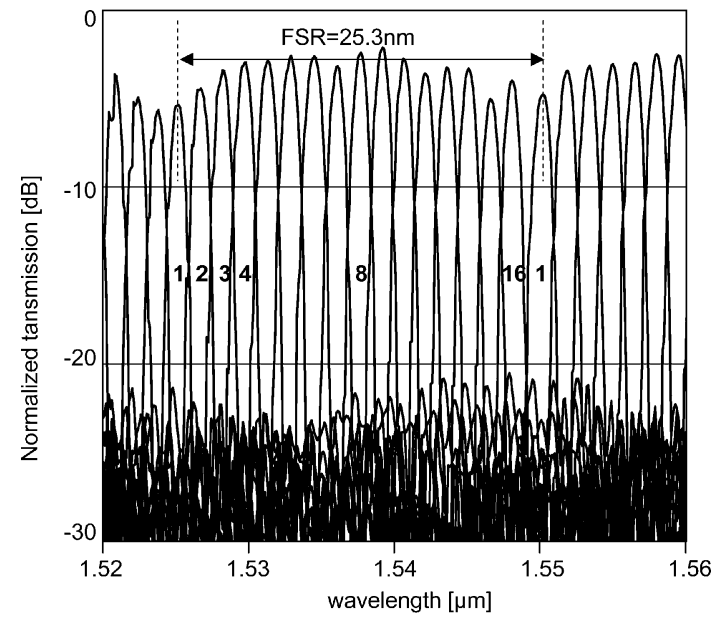

(b)

Fig. 5. Transmission spectrum of the 16 output ports of the AWG in Fig. 4. (a) Simulation. (b) Measurement.

\section{MACH-ZEHNDER LATTICE FILTERS}

An MZLF, sometimes called a cascaded Mach-Zehnder filter, consists of a series of Mach-Zehnder interferometers with a constant path-length difference but with varying coupling ratio. The coupling ratios can be optimized for the desired filter spectrum. For example, an MZLF can be designed to have Chebychevlike filter characteristic with low sidelobe levels. SOI photonicwire-based MZLFs have already been demonstrated by several authors [18], [19].

Fig. 6 shows an example of a five-stage MZLF, consisting of six directional couplers and five delay sections. Due to optical proximity effects during lithography [2], the isolated waveguides have a width of $565 \mathrm{~nm}$, while the waveguides in the directional couplers are only $535-\mathrm{nm}$ wide. The gap between the waveguides is $220 \mathrm{~nm}$.

Fig. 6(b) shows the transmission as a function of wavelength in the pass and the drop port, normalized to the transmission

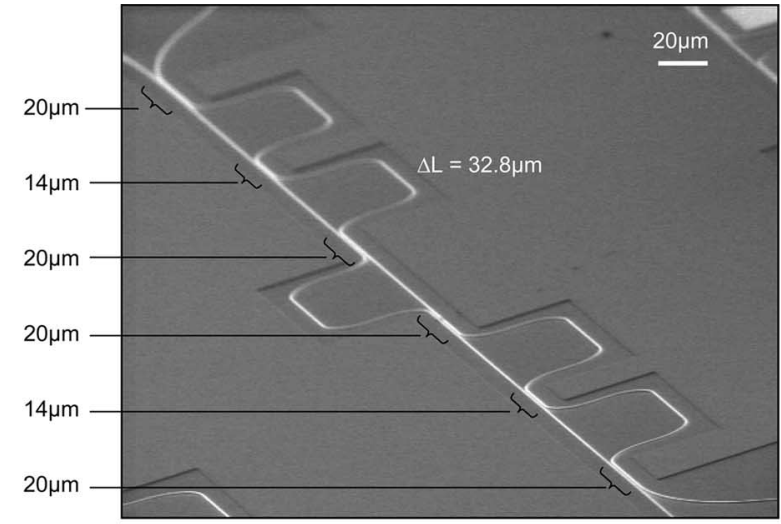

(a)

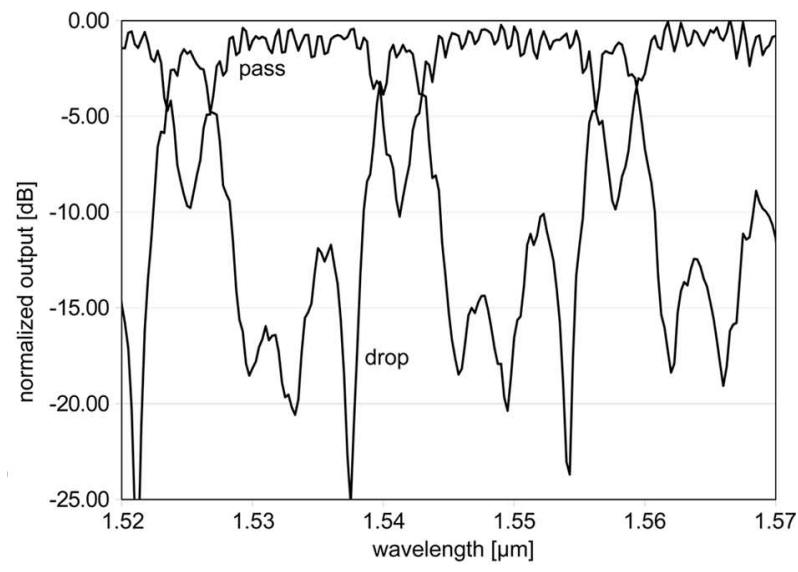

(b)

Fig. 6. Design of a five-stage MZLF. (a) Fabricated device. (b) Transmission spectrum of the pass and the drop port.

of a simple straight waveguide. The filter characteristic is welldefined with a bandwidth of $2.6 \mathrm{~nm}$, an FSR of $17 \mathrm{~nm}$, and a coupling efficiency of nearly $100 \%$. Crosstalk is still relatively large at $10 \mathrm{~dB}$, and is caused by nonoptimal coupling ratios and phase errors delay sections. Here too, improvements are possible using broadened photonic wires in the delay lines, and this will also result in a slightly larger device.

\section{RING AND RACETRACK RESONATORS}

\section{A. Single-Channel Drop Filters}

Ring and racetrack resonators can be used as a building block for densely integrated wavelength-selective filters. In photonic wires, the sharp bends allow for very compact rings with the very large FSR needed for add-drop filters. However, a reliable fabrication of such devices is challenging. We have demonstrated ring resonators with a radius between 1 and $8 \mu \mathrm{m}$, which are laterally coupled to two waveguides using directional couplers. To achieve low crosstalk and high finesse, we explored a variety of coupling geometries.

A circular ring resonator is shown in Fig. 7. The spacing in the directional coupler sections is limited to a minimum of $200 \mathrm{~nm}$ due to lithographic constraints. Note that the coupling section is quite short due to the curvature of the ring; therefore, 


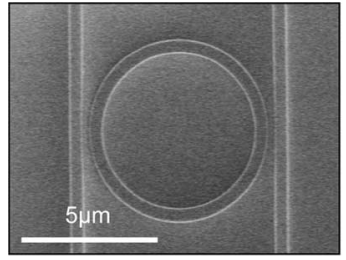

(a)

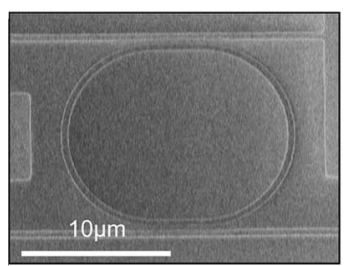

(c)

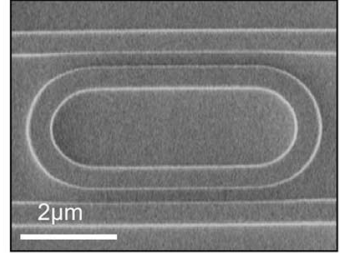

(b)

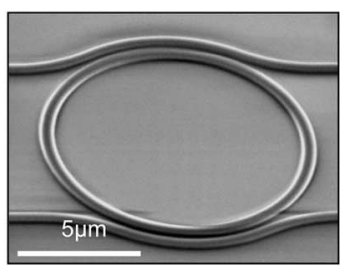

(d)
Fig. 7. Ring and racetrack resonators. (a) Circular ring resonator with a $3-\mu \mathrm{m}$ bend radius. (b) Racetrack resonator with a $1-\mu \mathrm{m}$ bend radius and a 3- $\mu \mathrm{m}$ straight coupling section. (c) Racetrack resonator with a $6-\mu \mathrm{m}$ bend and a 3- $\mu \mathrm{m}$ coupling section. (d) Bend-coupled ring resonator with a 5- $\mu \mathrm{m}$ bend radius.

the coupling efficiency is rather low. This is obvious from the transmission spectrum in Fig. 8(a) for a ring with 5- $\mu$ m radius. Even at resonance, the power in the pass port is still much larger than that in the drop waveguide.

To improve the coupling efficiency, one can use a straight coupling section, a so-called racetrack configuration [Fig. 7(b) and (c)]. With only a $1-\mu \mathrm{m}$ radius, a $Q$ of around 2100 and an FSR in excess of $40 \mathrm{~nm}$ are obtained, but the drop efficiency is low. The low $Q$ can be attributed to bend losses in the photonic wire. For larger radii, the losses decrease quickly. Fig. 8(b) shows transmission spectra of a racetrack with a 5- $\mu$ m radius and a 6.28- $\mu$ m coupling section. Larger coupling leads to a high adddrop extinction ratio of $-20 \mathrm{~dB}$ and $50 \%-70 \%$ drop efficiency with $Q$ factor still over 3000 . Due to the larger resonator, the FSR is reduced to approximately $12 \mathrm{~nm}$. For racetracks with radii down to $2 \mu \mathrm{m}$, we have measured $Q$-values up to 12000 . For even smaller bends, radiation losses become dominant and the $Q$ drops rapidly.

Because of the straight coupling section, the FSR of a racetrack resonator is limited. To improve the coupling efficiency of a ring without adding length to the resonator, one can use a bent waveguide for coupling [20] [Fig. 7(c)]. By tailoring the width of the resonator and the access waveguide, good phase matching is possible, and high coupling efficiencies can be achieved. The coupling can be adjusted by the angle of the coupling section. Fig. 8(c) demonstrates an extinction ratio (from -10 to -15 $\mathrm{dB}$ ) and drop efficiency in a resonator with a 5- $\mu \mathrm{m}$ radius.

Note that it is of utmost importance to control the feature sizes of the resonators very tightly. The coupling efficiency of the directional coupler sections depends strongly on spacing between the waveguides. Just as with the MZLF in Section VI, optical proximity effects can change the actual gap width, as well as the waveguide width in the coupler section with respect to the waveguide width in the isolated sections of the ring. As a small change in the waveguide width of $5 \mathrm{~nm}$ over the entire ring can lead to a shift in drop wavelength of about $15 \mathrm{~nm}$, a

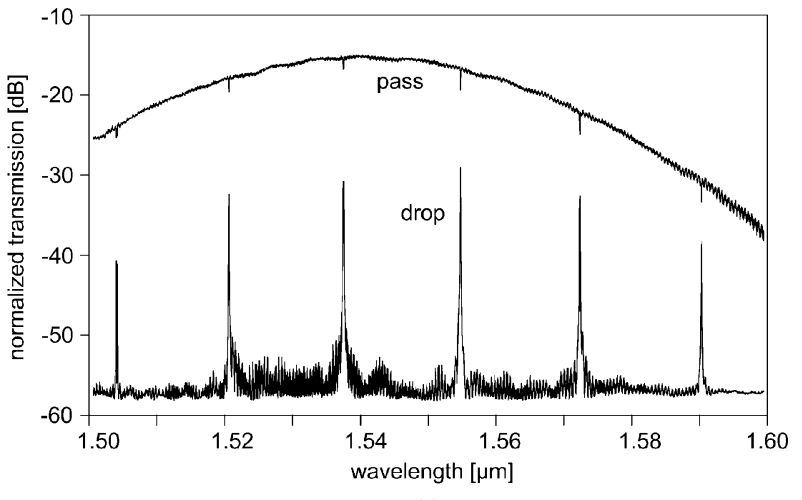

(a)

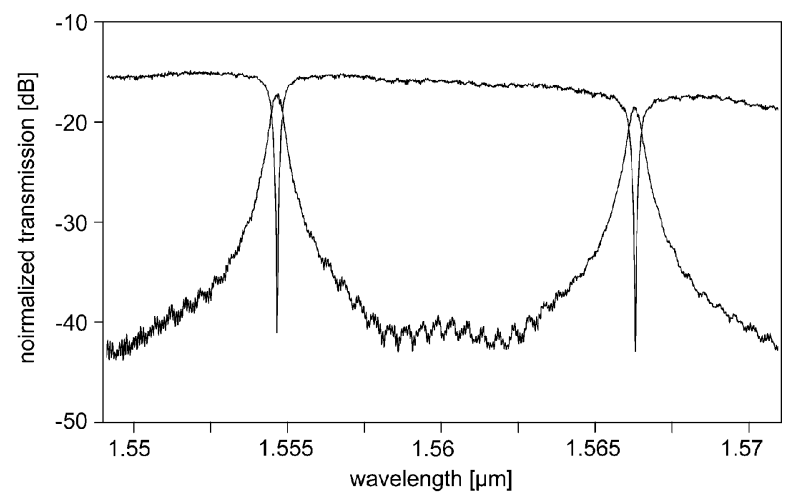

(b)

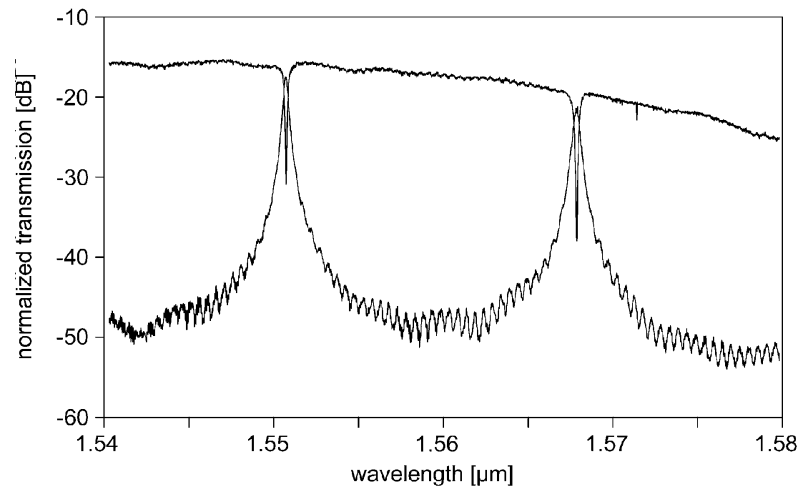

(c)

Fig. 8. Transmission spectra of drop and pass ports for resonators. (a) Ring with 5- $\mu \mathrm{m}$ radius. (b) Racetrack with $5-\mu \mathrm{m}$ bend radius and $6.28-\mu \mathrm{m}$ coupling section. (c) Ring with bent coupling section and a $5-\mu \mathrm{m}$ radius.

thorough optimization of the design and fabrication process is required.

\section{B. Four-Channel Add-Drop Filter With Racetrack Resonators}

Fig. 9 shows a four-channel wavelength add-drop filter using four racetrack resonators with different bend radii $(6,6.02$, 6.04 , and $6.08 \mu \mathrm{m})$. Note that with this approach not only the resonance wavelength but also the FSR vary. The transmission spectra are overlaid in Fig. 9(b). In this example, a large part of the FSR can be reached by changing the radius over only $80 \mathrm{~nm}$, but there is no linear relationship between the resonance wavelength and the change in radius. In our opinion, this is mainly because of the mask discretization, but other configurations will 


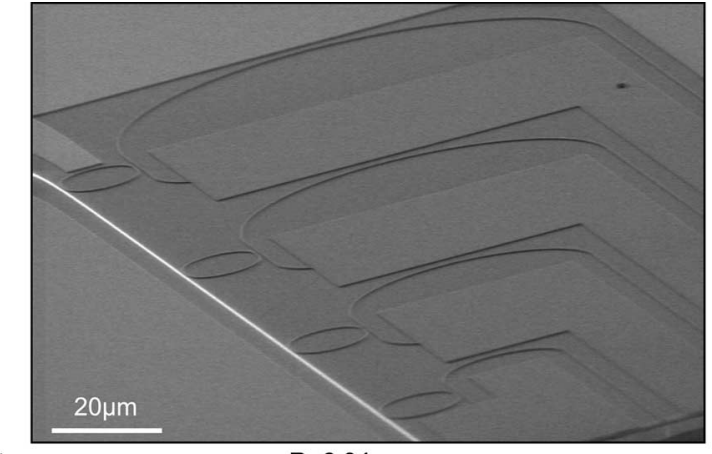

(a)

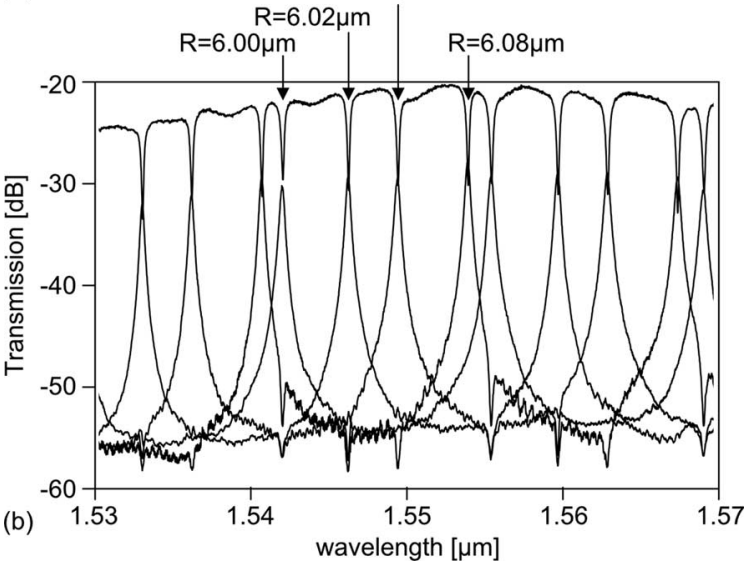

Fig. 9. (a) Four-channel demultiplexer using first-order racetrack resonators with different bend radius. (b) Overlaid transmission characteristics of the demultiplexer in the pass port and four drop ports.

be studied to avoid this problem and achieve better control of the wavelength spacing.

\section{CONCLUSION}

We have demonstrated very compact wavelength-selective functions in SOI photonic wire. These include AWGs, MZLFs, and ring resonators. While photonic wires allow for sharp bends and a high group index, they make wavelength-selective devices prone to phase noise. To alleviate this problem, we broadened the waveguides in straight sections with dramatic improvement in the crosstalk of an AWG. To improve the insertion loss in AWG star couplers, we employed a two-step etch process, reducing the lateral index contrast at the interface of a waveguide with the free-space section.

These improvements resulted in AWGs with areas of about $0.1 \mathrm{~mm}^{2}$ (for a 16-channel AWG with 1.6-nm channel spacing) with crosstalk levels of $-20 \mathrm{~dB}$ and an insertion loss of $-2.2 \mathrm{~dB}$ for the center channels. We have also shown a five-stage MZLF. The high crosstalk of $-10 \mathrm{~dB}$ can be improved by optimization of the coupling sections and by using broadened waveguides. Finally, we have shown compact ring resonators, including a four-channel add-drop multiplexer.

These devices show that it is possible to achieve a considerable reduction in size by using high-contrast photonic wires with strong confinement (and therefore sharp bends) and high group index (short delay lines). However, care is needed in interfer- ometric structures as the waveguides are prone to phase noise, though, this was remedied by using broadened waveguides.

\section{ACKNOWLEDGMENT}

The authors would like to thank J. Mees for mask design, J. Van Ongeval, J. Mentens, and the IMEC P-line operators for processing, and J. Schrauwen for SEM images.

\section{REFERENCES}

[1] W. Bogaerts, P. Bienstman, and R. Baets, "Scattering at sidewall roughness in photonic crystal slabs," Opt. Lett., vol. 28, no. 9, pp. 689-691, 2003.

[2] W. Bogaerts, R. Baets, P. Dumon, V. Wiaux, S. Beckx, D. Taillaert, B. Luyssaert, J. Van Campenhout, P. Bienstman, and D. Van Thourhout, "Nanophotonic waveguides in silicon-on-insulator fabricated with CMOS technology," J. Lightw. Technol., vol. 23, no. 1, pp. 401-412, Jan. 2005.

[3] W. Bogaerts, V. Wiaux, D. Taillaert, S. Beckx, B. Luyssaert, P. Bienstman, and R. Baets, "Fabrication of photonic crystals in silicon-on-insulator using 248-nm deep UV lithography," IEEE J. Sel. Topics Quantum Electron., vol. 8, no. 4, pp. 928-934, Jul.-Aug. 2002.

[4] D. Taillaert, W. Bogaerts, P. Bienstman, T. Krauss, P. Van Daele, I. Moerman, S. Verstuyft, K. De Mesel, and R. Baets, "An out-ofplane grating coupler for efficient butt-coupling between compact planar waveguides and single-mode fibers," IEEE J. Quantum Electron., vol. 38, no. 7, pp. 949-955, Jul. 2002.

[5] W. Bogaerts, D. Taillaert, B. Luyssaert, P. Dumon, J. Van Campenhout, P. Bienstman, D. Van Thourhout, R. Baets, V. Wiaux, and S. Beckx, "Basic structures for photonic integrated circuits in silicon-on-insulator," Opt. Express, vol. 12, no. 8, pp. 1583-1591, Apr. 2004.

[6] P. Dumon, W. Bogaerts, D. Van Thourhout, D. Taillaert, R. Baets, J. Wouters, S. Beckx, and P. Jaenen, "Compact wavelength router based on a silicon-on-insulator arrayed waveguide grating pigtailed to a fiber array," Opt. Express, vol. 14, no. 2, pp. 664-669, Jan. 2006.

[7] E. Dulkeith, F. Xia, L. Schares, W. M. J. Green, and Y. A. Vlasov, "Group index and group velocity dispersion in silicon-on-insulator photonic wires," Opt. Express, vol. 14, no. 9, pp. 3853-3863, May 2006.

[8] H. Gersen, T. Karle, R. Engelen, W. Bogaerts, J. Korterik, N. van Hulst, T. Krauss, and L. Kuipers, "Real-space observation of ultraslow light in photonic crystal waveguides," Phys. Rev. Lett., vol. 94, no. 7, p. 073903 , Feb. 2005.

[9] P. Dumon, W. Bogaerts, V. Wiaux, J. Wouters, S. Beckx, J. Van Campenhout, D. Taillaert, B. Luyssaert, P. Bienstman, D. Van Thourhout, and R. Baets, "Low-loss SOI photonic wires and ring resonators fabricated with deep UV lithography," IEEE Photon. Technol. Lett., vol. 16, no. 5, pp. 1328-1330, May 2004.

[10] S. J. McNab, N. Moll, and Y. A. Vlasov, "Ultra-low loss photonic integrated circuit with membrane-type photonic crystal waveguides," Opt. Express, vol. 11, no. 22, pp. 2927-2939, Nov. 2003.

[11] M. Notomi, A. Shinya, S. Mitsugi, E. Kuramochi, and H. Ryu, "Waveguides, resonators and their coupled elements in photonic crystal slabs," Opt. Express, vol. 12, no. 8, pp. 1551-1561, Apr. 2004.

[12] Y. A. Vlasov and S. McNab, "Losses in single-mode silicon-on-insulator strip waveguides and bends," Opt. Express, vol. 12, no. 8, pp. 1622-1631, Apr. 2004.

[13] P. Dumon, W. Bogaerts, D. Van Thourhout, D. Taillaert, V. Wiaux, S. Beckx, J. Wouters, and R. Baets, "Wavelength-selective components in SOI photonic wires fabricated with deep UV lithography," in Proc. Conf. Group IV Photonics, Hong Kong, Sep. 2004, p. WB5.

[14] C. R. Doerr, "Planar lightwave devices for WDH," in Optical Fiber Telecommunications IVA. San Diego, CA: Academic, 2002, ch. 9.

[15] T. Fukazawa, F. Ohno, and T. Baba, "Very compact arrayed-waveguidegrating demultiplexer using si photonic wire waveguides," Jpn. J. Appl. Phys., vol. 43, no. 5B, pp. L673-L675, 2004.

[16] K. Sasaki, F. Ohno, A. Motegi, and T. Baba, "Arrayed waveguide grating of $70 \times 60 \mu \mathrm{m}^{2}$ size based on Si photonic wire waveguides," Electron. Lett., vol. 41, no. 14, pp. 801-802, Jul. 2005.

[17] Y. Barbarin, X. J. M. Leijtens, E. A. J. M. Bente, C. M. Louzao, J. R. Kooiman, and M. K. Smit, "Extremely small AWG demultiplexer fabricated on InP by using a double-etch process," IEEE Photon. Technol. Lett., vol. 16, no. 11, pp. 2478-2480, Nov. 2004.

[18] K. Yamada, T. Shoji, T. Tsuchizawa, T. Watanabe, J. Takahashi, and S. Itabashi, "Silicon-wire-based ultrasmall lattice filters with wide free spectral ranges," Opt. Lett., vol. 28, no. 18, pp. 1663-1664, 2003. 
[19] T. Tsuchizawa, K. Yamada, H. Fukuda, T. Watanabe, J. Takahashi, M. Takahasha, T. Shoji, E. Tamechika, S. Itabashi, and H. Morita, "Microphotonics devices based on silicon microfabrication technology," IEEE J. Sel. Topics Quantum Electron., vol. 11, no. 1, pp. 232-240, Jan.-Feb. 2005.

[20] M. Chin and T. Ho, "Design and modeling of waveguide-coupled singlemode microring resonators," J. Lightw. Technol., vol. 16, no. 8, pp. 14331446, Aug. 1998.

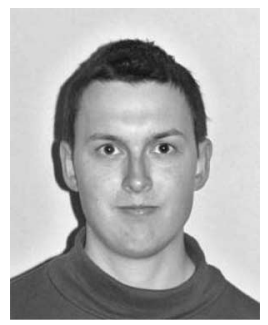

Wim Bogaerts (S'97-A'98-M'03) received the Graduate degree in applied physics from Ghent University, Ghent, Belgium, in 1998, and the Ph.D. degree from the Department of Information Technology (INTEC) and the Interuniversity Microelectronics Center (IMEC), Ghent University, in 2004.

He specializes in the modeling, design, and fabrication of nanophotonic components. He is currently engaged in coordinating the fabrication of nanophotonic components in silicon-on-insulator at the IMEC as a part of the European Network of Excellence

ePIXnet.

Dr. Bogaerts is a member of the IEEE Lasers and Electro-Optics Society and the Optical Society of America.

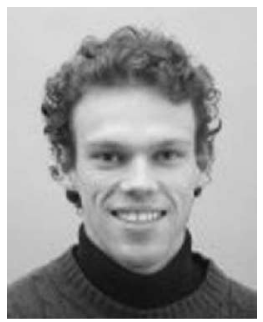

Pieter Dumon (S'02) received the Graduate degree in electrical engineering from Ghent University, Ghent, Belgium, in 2002, where he is currently working toward the $\mathrm{Ph} . \mathrm{D}$. degree in electrical engineering.

His current research interests include modeling, design, and fabrication of nanophotonic waveguides and structures for passive photonic integrated circuits.

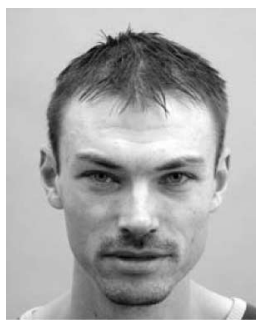

Dries Van Thourhout (M'98) received the Graduate degree in physical engineering and the Ph.D. degree in electrical engineering from Ghent University, Ghent, Belgium, in 1995 and 2000, respectively.

During 2000-2002, he was with Lucent Technologies, Bell Laboratories, Crawford Hill, NJ, where he was engaged in work on InPInGaAsP monolithically integrated devices. Since 2002, he has been with the Department of Information Technology (INTEC) at Ghent University. His current research interests include heterogeneous integration by wafer bonding, intrachip optical interconnect, and WDM devices.

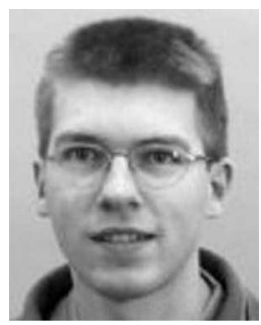

Dirk Taillaert (S'97-M'04) received the Graduate degree in electrical engineering from Ghent University, Ghent, Belgium, in 1999, where he is currently working toward the Ph.D. degree in electrical engineering.

$\mathrm{He}$ is engaged in work on waveguide-fiber interfaces. His current research interests include design, fabrication, and characterization of waveguides and components for micro/nanophotonics.

Mr. Taillaert is a member of the IEEE Lasers and Electro-Optics Society.

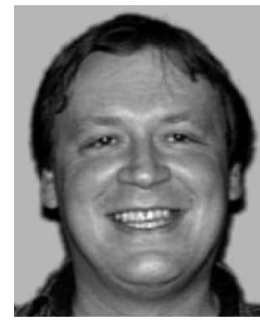

Patrick Jaenen received the Graduate degree in chemical engineering from the Technische Hogeschool DENAYER, Mechelen, Belgium, in 1984.

$\mathrm{He}$ is currently a Senior Litho Process Engineer at the Interuniversity Microelectronics Center, Leuven, Belgium, where he is working in the litho group on 248- and 193-nm process development.

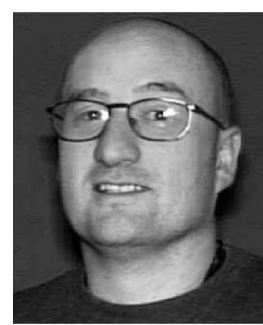

Johan Wouters joined the Interuniversity Microelectronics Center, Leuven, Belgium, as Etch and Oven Operator, in 1988 . He is currently a Dry Etch Process Developer Assistant.

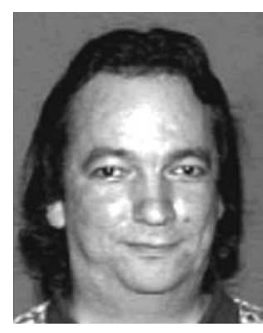

Stephan Beckx received the Ph.D. degree in coordination chemistry from the Catholic University of Leuven, Leuven, Belgium, in 1993.

In 1994, he joined the Interuniversity Microelectronics Center (IMEC), Leuven, where he is currently a Senior Process Engineer in the AMPS Department. $\mathrm{He}$ is responsible for the follow-up of advanced frontend-of-line etch development and related engineering.

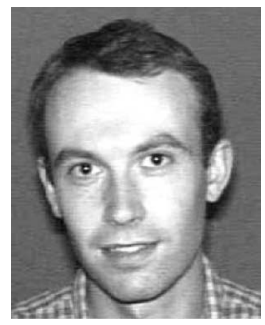

Vincent Wiaux received the M.S. and Ph.D. degrees in physics from the Catholic University of Leuven, Leuven, Belgium, in 1995 and 1999, respectively.

Since 1999, he has been with the Interuniversity Microelectronics Center, Leuven, where he is engaged in the application of resolution enhancement techniques to low-k1 back-end imaging.

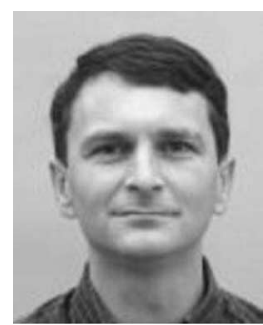

Roel G. Baets (M'88-SM'96) received the Graduate degree from Ghent University, Ghent, Belgium, in 1980, and the M.Sc. degree from Stanford University, Stanford, CA, in 1981, and the Ph.D. degree from Ghent University, in 1984, all in electrical engineering.

Since 1981, he has been with the Department of Information Technology (INTEC) of Ghent University. Since 1989, he has been a Professor in the Faculty of Engineering. During 1990-1994, he was also a parttime Professor at the Technical University of Delft, Delft, The Netherlands. He leads the Photonics Group at Ghent UniversityINTEC, an associated laboratory of the Interuniversity Microelectronics Center (IMEC), working on integrated photonics for optical communications, interconnects, and sensing. He is the author or coauthor of more than 300 publications and conference papers and is the holder of ten patents. His current research interests include III-V semiconductor laser diodes, passive guided-wave devices, photonic ICs, and microoptics.

Prof. Baets has been a member of the program committees of OFC, ECOC, IEEE Semiconductor Laser Conference, ESSDERC, CLEO-Europe, and the ECIO. During 1999-2001, he was the Chairman of the IEEE LEOS Benelux Chapter. He is a member of the Optical Society of America, IEEE Lasers and Electro-Optics Society, SPIE, and the Flemish Engineers Association. 